\title{
edoc
}

Institutional Repository of the University of Basel

University Library

Schoenbeinstrasse 18-20

CH-4056 Basel, Switzerland

http://edoc.unibas.ch/

\section{Cortisol awakening response in infants during the first six postnatal months and its relation to birth outcome}

\author{
Marion Tegethoff, PhD, Nicole Knierzinger, MSc, Andrea H. Meyer, PhD, \\ Gunther Meinlschmidt, $\mathrm{PhD}$
}

Posted at edoc, University of Basel

Official URL: http://edoc.unibas.ch/dok/A6008534

Originally published as:

Tegethoff, M., Knierzinger, N., Meyer, A. H., \& Meinlschmidt, G. (2013). Cortisol awakening response in infants during the first six postnatal months and its relation to birth outcome. Psychoneuroendocrinology, 38, 5, 629-637.




Tegethoff et al.: CAR in infants

Cortisol awakening response in infants during the first six postnatal months and its relation to birth outcome

\section{${ }^{1}$ Marion Tegethoff, $\mathrm{PhD}^{2}$, ${ }^{1}$ Nicole Knierzinger, $\mathrm{MSc}^{3}$, Andrea H. Meyer, $\mathrm{PhD}^{3,4}$, Gunther Meinlschmidt, PhD ${ }^{* 3,5,6}$}

${ }^{1}$ both authors contributed equally

${ }^{2}$ Division of Clinical Psychology and Psychiatry, Department of Psychology, University of Basel, Missionsstrasse 60/62, CH-4055 Basel, Switzerland

${ }^{3}$ Division of Clinical Psychology and Epidemiology, Department of Psychology, University of Basel, Missionsstrasse 60/62, CH-4055 Basel, Switzerland

${ }^{4}$ Section of Applied Statistics in Life Sciences, Division of Clinical Psychology and Epidemiology, Department of Psychology, University of Basel, Missionsstrasse 60/62, CH4055 Basel, Switzerland

${ }^{5}$ Research Department of Psychobiology, Psychosomatics, and Psychotherapy, Clinic of Psychosomatic Medicine and Psychotherapy, LWL University Hospital, Ruhr-University

Bochum, Alexandrinenstrasse 1-3, D-44791 Bochum, Germany

${ }^{6}$ National Centre of Competence in Research "Swiss Etiological Study of Adjustment and Mental Health (sesam)", Missionsstrasse 60/62, CH-4055, Switzerland

*Corresponding author and request for reprints:

Gunther Meinlschmidt, Ph.D.

Professor and Director of the Research Department of Psychobiology, Psychosomatics, and Psychotherapy; Clinic of Psychosomatic Medicine and Psychotherapy,

LWL University Hospital, Ruhr-University Bochum

Alexandrinenstrasse 1-3, D-44791 Bochum, Germany

Phone: ++4923450773173

Fax: ++49 23450773111

Email: Gunther.Meinlschmidt@rub.de 
Tegethoff et al.: CAR in infants

Running title: CAR in infants

Word count (excluding abstract, figure captions, and references): 3861

Number of tables: 1 ; Number of figures: 2

\section{Second Revision for Psychoneuroendocrinology}

\section{Summary}

Context: The rise of cortisol concentrations after awakening is well documented in adults and children and commonly used as easily accessible marker of hypothalamic-pituitary-adrenal (HPA) reactivity.

Objective: The purpose of this study was to demonstrate the existence of a salivary cortisol awakening response (CAR) in infants, and to estimate its association with birth outcome.

Setting: The study was conducted in the general community.

Participants: Healthy infants up to six months age ( $\mathrm{N}=64)$.

Main outcome measures: Mothers were instructed to collect their infant's saliva immediately and 30 min after awakening on two days within 45 days, irrespective of awakening time. Information on birth outcome was collected from medical records and questionnaires.

Results: Linear mixed models analysis revealed a significant rise of infant salivary cortisol concentrations within 30 minutes after awakening $\left(b=0.128, \mathrm{SE}=0.024, t_{61}=5.31, p<0.001\right)$, which was quite stable across the two sampling days $(r=0.40, p=0.002)$. The infant CAR was predicted by length of gestation $\left(t_{61}=2.43, p=0.018\right)$.

Conclusions: The current data demonstrate the existence of a CAR in infants as early as during the first six postnatal months; its relationship with length of gestation supports its usefulness for questions related to developmental neuroscience. Therefore, the infant CAR emerges as non-invasive biomarker of HPA axis dynamics at this early stage of life, with relevance for future research and potential clinical applications. 
Tegethoff et al.: CAR in infants

Keywords: birth weight; cortisol awakening response (CAR); developmental programming; hypothalamic-pituitary-adrenal (HPA) axis; length of gestation; newborns 


\section{Introduction}

The hypothalamic-pituitary-adrenal (HPA) axis is a major endocrine system and regulates the secretion of glucocorticoids, such as cortisol. Glucocorticoids are important regulators of a wide range of physiological processes, including lipid, protein, and carbohydrate metabolism, and of cardiovascular, neurobiological, and immunological function. One major function of glucocorticoids is to maintain homeostasis and to enable coping with and adaptation to stressful events (Sapolsky et al., 2000). Cortisol is released in response to biochemical and psychosocial stimuli (Dickerson and Kemeny, 2004), and spontaneously throughout the day (Weitzman et al., 1971). The HPA axis develops early during fetal life and becomes functional around mid-gestation (Mesiano and Jaffe, 1997). HPA axis activity and reactivity is susceptible to a variety of factors already as early as during the prenatal phase (Matthews, 2002; Tegethoff et al., 2009). This phenomenon has been described as 'fetal programming' (Phillips and Jones, 2006), a specification of the more general concept of 'programming', which describes the process by which a stimulus or insult at a critical stage of development has lasting or lifelong significance (Lucas, 1991). The well-documented circadian rhythm of cortisol emerges within the first months postpartum (Price et al., 1983; Santiago et al., 1996; Antonini et al., 2000; Groschl et al., 2003).

While secretory activity is in general relatively low at night, cortisol levels slowly start to rise during the second half of the night, with a sharp increase upon awakening, peaking in saliva at about 30 min after awakening (Wilhelm et al., 2007). During this peak, cortisol levels increase on group average by between $50 \%$ and $160 \%$, with a minor fraction of subjects displaying a 'negative CAR' (i.e. decrease of cortisol concentrations after awakening) (Van Cauter et al., 1996; Wust et al., 2000; Edwards et al., 2001; Kudielka et al., 2007; Wilhelm et al., 2007; Dockray et al., 2008). Pruessner et al. (1997) were the first to establish the cortisol awakening response (CAR) as a reliable marker of adrenocortical activity (Hellhammer et al., 2009). Further research demonstrated that the CAR represents a distinctive part of the diurnal 
cortisol cycle, independent of the underlying basal activity and stimulated by extra-HPA pathways (Edwards et al., 2001; Clow et al., 2010). Although the CAR has been well documented in adults and children of various age groups ranging from 4 to 75 years (Kudielka and Kirschbaum, 2003), knowledge on the existence of the CAR in younger infants is hitherto lacking. However, there is ample evidence on developmental changes in circadian regulation and HPA activity and reactivity during infancy. With regard to the development of a circadian rhythm in HPA activity, previous studies have suggested that a circadian regulation of the HPA axis is already present at 12 months of age, but continues to mature into the third year, corresponding to changes in behavioral development, including sleeping patterns (Watamura et al., 2004), with reduced total sleep duration, less night wakings and less daytime naps related to increasing age during infancy and beyond (Galland et al., 2012). Notably, overall, baseline salivary cortisol concentrations tended to decrease between one and three years of age (Watamura et al., 2004). While psychological stressors do not consistently provoke a cortisol reaction in infants, in response to physical stressors, the infant HPA axis mostly reacts with a moderate cortisol reaction during the postpartal period, with decreasing reactivity effect sizes with increasing infant age (Gunnar et al., 1996; Gunnar et al., 2009; Jansen et al., 2010).

The physiological role of the CAR is as yet unclear but there is evidence that the CAR might play a metabolic and immunological role in the transition from sleep to wakeful state, and it has been associated with different psychosocial factors (Pruessner et al., 1997; Chida and Steptoe, 2009; Fries et al., 2009). Importantly, changes in the CAR have been described in patients with mental and somatic disorders (Clow et al., 2004; Kudielka and Wust, 2010), including coronary artery disease, systemic hypertension, respiratory disorders, obesity, functional gastrointestinal disorders, bipolar illness, depressive symptoms, borderline personality disorder, chronic fatigue, and posttraumatic stress disorder, indicating the clinical relevance of the CAR. Furthermore, changes in HPA axis function have been related to early 
postnatal adversities (e.g. Meinlschmidt and Heim, 2005) and prenatal factors. In a number of studies, low birth weight or being small for gestational age was related to alterations in HPA axis (re-)activity during infancy, childhood, and later life (Phillips et al., 2000; Reynolds et al., 2001; Wust et al., 2005; Jones et al., 2006; Osterholm et al., 2012). However, the time of onset of such HPA alterations and specifically the relationship between birth outcome and the CAR in infants remains as yet unknown. Such information might help elucidating the potential role of the HPA axis in mediating the association between birth outcome and lifelong disease (see Osterholm et al., 2012). Birth outcome, used as markers of intrauterine development and linked with intrauterine adversities in a large number of studies (e.g. Tegethoff et al., 2010a, b), has been associated with stress-related cerebral activity (Jones et al., 2011) and various diseases across life, including coronary heart disease, hypertension, and diabetes (Osmond and Barker, 2000).

The main objective of the present study was to show that the CAR is detectable in infants during the first six postnatal months. Second, to provide proof-of-concept for the usefulness of the CAR in infants as research tool, we determined the relationship of the infant CAR with birth outcomes.

\section{Methods}

\section{Overall study procedure}

To recruit infants, mothers were contacted via the University Women's Hospital of Basel or via birth announcements within the first three weeks after parturition. Maternal or infant endocrine disorders, genetic anomalies, birth defects and postnatal steroid treatment of the newborn were exclusion criteria. The study was conducted according to the declaration of Helsinki and the local ethics committee approved the study. All mothers gave written informed consent. 


\section{Instruments}

Information on demographics, sleeping, feeding, and saliva collection was obtained by questionnaires. Information on birth characteristics was obtained by medical records or questionnaires. We calculated sex-specific birth weight percentiles, using previously published birth weight tables (Oken et al., 2003).

\section{Saliva samples}

Each mother was instructed to collect her infant's saliva during the first six months after parturition by using eyespears sampling devices (Visispears, Becton Dickinson AG, Basel, Switzerland). Compared to cotton salivettes which were shown to reduce the concentration of cortisol, eyespears do not influence cortisol concentrations and show less interference in the rank ordering of cortisol (Strazdins et al., 2005). Moreover, eyespears have a smaller cotton swab and they can be held during sampling with a grasp, making them more suitable for use in newborns (Strazdins et al., 2005). Saliva was collected immediately after awakening from at least 30 minutes of sleep and 30 minutes later, on two different days (Hellhammer et al., 2007) within 45 days. With duration of at least 30 minutes of sleep, we expected to cover daytime naps of average duration during the first postnatal months (Tikotzky et al., 2010; Galland et al., 2012). Mothers were instructed neither to feed or breastfeed their infants after awakening before taking the first sample nor during the 30 minutes between the samplings, as milk contamination can lead to false high cortisol results (Magnano et al., 1989). Collection of the first saliva sample occurred between $0600 \mathrm{~h}$ and $1200 \mathrm{~h}$ at 61 samplings, between 1200 $\mathrm{h}$ and $1800 \mathrm{~h}$ at 45 samplings, between $1800 \mathrm{~h}$ and $2400 \mathrm{~h}$ at 14 samplings, and never between $0000 \mathrm{~h}$ and $0600 \mathrm{~h}$ (information missing for one sample). All samples were stored at $-20^{\circ} \mathrm{C}$ until assayed. Samples were thawed and centrifuged at $1000 \mathrm{rpm}$ for 10 minutes. Free cortisol concentrations were determined by an enzyme-linked immunosorbent assay (IBL International GmbH, Hamburg, Germany) according to standard procedures. 


\section{Participants}

We recruited a total of 107 mother-infant pairs. We considered only those subjects in whom salivary cortisol concentrations could be determined in both samples on at least one of the two sampling days, not taking into account samples from days when only one cortisol concentration could be determined. Moreover, a second sampling day had to occur not later than 45 days after the first, for the respective cortisol values from the second day to be considered. Hence, of the initial 107 mother-infant pairs, 64 (60\%) were included in the study (for details, see Figure 1) so that a total of 242 saliva samples was available for the study (out of these 64 infants, 57 infants contributed 4 and 7 infants contributed 2 saliva samples from which we could determine cortisol concentrations). Characteristics of the study sample are depicted in Table 1. None of the newborns had low birth weight (i.e. birth weight below 2500 g); six newborns were small for gestational age at birth (i.e. below the 10th birth-weight percentile) and three were born preterm (i.e. birth before the $37^{\text {th }}$ week of gestation).

\section{Statistical analyses}

We performed descriptive analyses of maternal and infant demographic and clinical baseline characteristics by calculating frequencies and percentages of the discrete variables. We calculated means and standard deviations for symmetrically distributed variables and medians and ranges for variables with non-symmetrical distributions, as distinguished by visual inspection of data plots.

A linear mixed model analysis was performed to calculate the influence of sampling day (day 1 and day 2) and sampling time (at awakening, 30 minutes after awakening), as well as their interaction, on the outcome variable infant salivary cortisol concentrations. Mixed models account for the dependence between repeated intra-individual measurements. The outcome variable was double-ln-transformed to meet assumptions of homogeneity of variances and 
normality of distribution. We dealt with missing data by restricting the analyses to certain participants and samples (see section 'participants'). We estimated the stability of the CAR by calculating Pearson's product-moment correlation coefficient. To verify whether the CAR depended on feeting between sampling, we repeated the respective analysis, including only samples of days when infants were not fed between the samplings

On 31 out of 121 sampling days (23.2\%), infants had been fed between samplings (28 times breast fed and 3 times formula fed), which was against the instruction. Therefore, to control for potential bias by milk (Magnano et al., 1989), and also for potential influences by the time of awakening (Clow et al., 2004) and sleep problems (Antonini et al., 2000; White et al., 2000), we repeated the mixed model analysis including 'feeding between samplings' (dichotomous variable), 'time of awakening' (continuous variable), and 'sleep problems' (repeatedly waking up again, difficulty to fall back asleep, inconsolability; continuous variable (score: 3-12)), as well as the interactions of these variables with sampling time, as covariates. For the additional analyses, we report the interactions between the variables of interest and the sampling time, as these interactions reflect the relationships with the CAR.

To identify a potential role of the infant's sex (Kudielka and Kirschbaum, 2005) and age at the time of sampling (Clow et al., 2004), we repeated the baseline mixed model analysis including sex (dichotomous variable) and 'age at time of sampling' (continuous variable), and the interactions of these variables with sampling time, as predictors.

Furthermore, to estimate the association of birth outcome and infant CAR, we repeated the baseline mixed model analysis including the interaction of sampling time with birth weight percentiles and length of gestation, respectively, as these variables are routine obstetric indicators of intrauterine development and well-established predictor of disease throughout life (Osmond and Barker, 2000; Wang et al., 2004), as predictors.

The level of significance was set at 0.05 for all analyses, and tests were two-tailed. SPSS software (version 17.0) was used for statistical analyses. 


\section{Results}

\section{Cortisol awakening response}

Mixed models analysis revealed a significant rise of infant salivary cortisol concentrations within 30 minutes after awakening $\left(\mathrm{b}=0.128, \mathrm{SE}=0.024, t_{61}=5.26, p<0.001\right)$, while the mean cortisol concentrations $\left(\mathrm{b}=0.011, \mathrm{SE}=0.020, t_{61}=-0.55, p=0.59\right)$ and the cortisol increase after awakening $\left(\mathrm{b}=0.040, \mathrm{SE}=0.032, t_{58}=1.24, p=0.22\right)$ did not significantly differ between the two sampling days, as shown in Figure 2. The change in cortisol concentrations within 30 minutes after awakening was quite stable across the two sampling days $(r=0.40, p=0.002)$. When stratifying the sample according to infants' age at time of sampling (younger group: age at sampling between 0 and 60 days ( $n=61)$; older group: age at sampling between 61 and 180 days $(n=60))$, the magnitude of the change in cortisol concentrations within 30 minutes after awakening was $0.32 \mu \mathrm{g} / \mathrm{dL}(\mathrm{SE}=0.082)$ and $0.27 \mu \mathrm{g} / \mathrm{dL}(\mathrm{SE}=0.063)$, respectively. The stability in the change in cortisol concentrations within 30 minutes after awakening across the two sampling days did not depend on the time lag between the two sampling days $(p>0.05)$. Repeating the analysis, including only samples of days when infants were not fed between the samplings $(n=53)$ led to similar results regarding the CAR $\left(t_{51}=4.44, p<0.001\right)$. Notably, not on all days, cortisol increased after awakening: Based on observed values, the cortisol concentration increased in 76 of $121(63 \%)$ days and decreased in $45(37 \%)$ days from sample one to sample two.

Feeding between sampling (interaction feeding $\mathrm{x}$ sampling time: $t_{125}=1.10, p=0.27$ ), time of awakening (interaction time of awakening x sampling time: $t_{125}=1.90, p=0.06$ ), and sleep problems (interaction sleep problems x samplig time: $t_{52}=0.42, p=0.67$ ) did not influence the change in cortisol concentrations across sampling times. Neither infant sex (interaction sex x sampling time: $t_{61}<0.01, p>0.99$ ) nor infant age at time of sampling (interaction age at time of sampling x sampling time: $t_{57}=0.22, p=0.83$ ) were associated with changes in infant salivary 
cortisol concentrations across sampling times. Changes in cortisol concentrations between awakening and 30 minutes after awakening varied with length of gestation (interaction length of gestation $\mathrm{x}$ sampling time: $t_{61}=2.43, p=0.018$ ), but not birth weight percentiles (interaction birth weight percentiles $\mathrm{x}$ sampling time: $\left.t_{60}=1.75, p=0.085\right)$. Whether information on birth outcomes was derived from medical documentation $(n=48,75 \%)$ or not, did not affect the results.

Furthermore, changes in cortisol concentrations between awakening and 30 minutes after awakening did not vary with general feeding style, i.e. whether the mother in general was breastfeeding (59 out of 64 mothers) or not (interaction breast feeding x sampling time: $t_{58}=-$ $0.94, p=0.35)$.

\section{Discussion}

In this study, we showed that a salivary CAR is detectable in infants as early as during the first six postnatal months. The CAR was relatively stable across the two sampling days and independent of awakening time and feeding between samplings. While longer length of gestation predicted an increased CAR during the first six postnatal months, the CAR did not vary with gender and infant age.

The findings of our study are in line with and add to the well-documented existence of a CAR in adults and older children, as demonstrated in previous studies (Pruessner et al., 1997; Edwards et al., 2001; Kudielka and Kirschbaum, 2003), and the stability of the CAR across the two sampling days was roughly comparable to previously reported association estimates calculated across four days (Hellhammer et al., 2007). The early emergence of the CAR is in accordance with previous findings that infants show a relatively strong HPA response to acute stressors compared to older children (Gunnar et al., 2009; Jansen et al., 2010; see also introduction section). 
The CAR seems to constitute a distinct part of the fluctuating cortisol secretion throughout the day (Pruessner et al., 1997), which primarily reflects phasic psychophysiological processes specific to the sleep-wake transition (Wilhelm et al., 2007). Even though there has been some controversy, our finding that results were not biased by the time of awakening is in line with the majority of studies that did not observe an association between the time of awakening and the CAR (Clow et al., 2004). Moreover, as the circadian variation of the HPA axis is less pronounced during the postpartum period as compared to adulthood (Watamura et al., 2004), circadian influences on the CAR in infants are less likely than in adults.

While gender differences in stress responsiveness are well documented in adults (Kajantie and Phillips, 2006), there is a lack of consensus whether the CAR is gender-specific in adults (Pruessner et al., 1997; Wust et al., 2000; Kudielka and Kirschbaum, 2003) and whether HPA axis activity is gender-specific in children (Kudielka and Kirschbaum, 2005). Notably, there is some evidence that gender-specificity of adrenocortical activity evolves during pubertal development and is absent in young children (Kiess et al., 1995; Groschl et al., 2003; Netherton et al., 2004; Tegethoff et al., 2011b). In line with this, we did not reveal gender differences in the CAR of infants.

Even though we did not detect any association between age and the CAR, future studies should address the question of whether the CAR varies with age during the first six months in more detail, by systematically assessing infants of different ages.

Our finding that length of gestation, but not birth weight percentiles, was associated with the CAR lends support to the hypothesis that a more pronounced CAR during early infancy is indicative of a more mature HPA axis, which would be interesting to scrutinize in future studies. Previous data on the association between birth outcome and the CAR is sparse: Two studies focused on the CAR in adults and its relation to body size at birth but did not reveal an association between these variables (Ward et al., 2004; Gustafsson et al., 2010), lending support to the hypothesis that the infant rather than the adult CAR is affected by intrauterine 
disturbances, and that birth outcome-related changes in the CAR might disappear or be compensated over time. This would be in line with a hypothesis put forth earlier, suggesting that the HPA axis might switch from a hyperactive to a hypoactive state (Buske-Kirschbaum et al., 2004).

Beyond the association between length of gestation and the CAR in early infancy, there is growing evidence that length of gestation, as well as other factors, such as maternal stress or intrauterine exposure to glucocorticoids, play a role in HPA dysfunction in later life and disease development (e.g. Phillips, 2007; Tegethoff et al., 2011a; Tollenaar et al., 2011; O'Connor et al., 2012; Tegethoff et al., 2012). Our finding generally supports the idea that birth outcomes are linked to later HPA activity. However, it is difficult to compare our findings to most previous studies because different biological fluids (blood serum, urine, saliva) and target variables (24-h profile, single morning samples, pharmacological challenge) were used to study the relationship between birth outcome and HPA activity. The early existence of HPA axis changes in relation to birth outcomes suggests a potential causal role of HPA disturbances in onset of diseases related to early adversities, which is as yet a matter of debate (Jessop and Turner-Cobb, 2008).

The specific mechanisms underlying the relationship between length of gestation and infant CAR are as yet unknown, but it seems reasonable that mechanisms involved in intrauterine HPA programming, such as epigenetic modifications at the glucocorticoid receptor gene, or general maturation processes might play a role (Matthews, 2002; Meaney et al., 2007).

Our results introduce the salivary CAR as a reliable, noninvasive, ambulatory tool to assess HPA reactivity in newborns and infants. As yet, studying adrenocortical reactivity in young children has strongly relied on stress paradigms, for example maternal separation (Goldberg et al., 2003) or blood draws and inoculation (Felt et al., 2000), even though such approaches face logistic (e.g. laboratory based assessment required) and ethical limitations and there is 
still some difficulty in understanding why some stressors successfully produce elevated cortisol levels whereas others do not (Gunnar et al., 2009; Jansen et al., 2010).

Strengths of the study include the repeated saliva collection across two days (Hellhammer et al., 2007), the use of a previously validated sampling method (Strazdins et al., 2005), the consideration of a potential influence of milk and time of awakening (Magnano et al., 1989), and accounting for the dependence between repeated intra-individual measurements by performing mixed model analyses.

There are also limitations to our study. First, our sample might not be representative of the general population, e.g. regarding the mothers' age. The Swiss Annual Population Statistics (ESPOP) states that the average age of maternity was 30.8 years in 2007 . With an average age of 33.8 years, the mothers in our study population were slightly older. Another limitation is the lack of control over the participants' compliance. We did not use electronic monitoring, including evaluation of infants' sleeping patterns. Even though mothers were instructed not to feed or breastfeed their babies before and between saliva samplings, some still did so. Therefore, this instruction needs to be revised in future studies. However, controlling for feeding between saliva samplings revealed that feeding did not influence the CAR in infants. Moreover, we did not collect information on sleep duration, and hence could not estimate whether sleep duration before awakening was related to the magnitude of the CAR. Furthermore, we did not collect information on maternal passive exposure to tobacco smoke, which has been reported to be associated with changes in birth outcomes. Besides that, even though we instructed the mothers to refer to written birth documentation, when providing information on birth outcome, we cannot finally rule out recall bias, as we did not collect all information on birth outcome directly from hospital birth records. Finally, age at assessment as well as number of days between assessments substantially varied.

Future studies should further address the clinical relevance of the infant CAR and, for example, shed some light on its potential relation with pediatric diseases by carefully 
monitoring the CAR in clinical pediatric samples and prospectively following-up infant cohorts. Such studies might help to clarify the role of the CAR as psychobiological disease biomarker and provide a better basis for its potential use as a diagnostic tool.

Moreover, future studies may use the infant CAR to explore the existence of a stress hyporesponsive period (SHRP) in humans. In rodents, this period is characterized by a reduced HPA axis response to stressors, lasting from about postnatal day 4 to 14 (Schapiro et al., 1962; Schmidt et al., 2003). Research has not yet resolved the question of whether or not such a period of HPA hypo-responsiveness also exists in humans (Lupien et al., 2009). Therefore, future studies should scrutinize the SHRP using repeated sampling, for example in weekly intervals, within the first months after birth. As our sample only includes a few infants born preterm or small for gestational age, future studies are needed to estimate the magnitude of the associations between being born preterm or small for gestational age and the CAR.

Finally, we cannot conclude, whether the process of per se awakening or other factors related to awakening (such us not being fed immediately after awakening or the procedure of the first saliva collection) have led to the CAR. While we did not detect an association between feeding and the magnitude of the CAR, future studies should scrutinize the nature of the CAR in infants.

In summary, the current data demonstrate the existence of a CAR as early as during the first six postnatal months; its relationship with length of gestation, a well-established predictor of a wide range of diseases throughout life, supports its relevance as research tool in developmental psychobiology. Therefore, the infant CAR may be a non-invasive biomarker of HPA axis dynamics at this early stage of life, with relevance for future research and potentially for clinical practice. 
Tegethoff et al.: CAR in infants

\section{References}

Antonini, S.R., Jorge, S.M., Moreira, A.C., 2000. The emergence of salivary cortisol circadian rhythm and its relationship to sleep activity in preterm infants. Clin Endocrinol (Oxf) 52, 423-426.

Buske-Kirschbaum, A., Fischbach, S., Rauh, W., Hanker, J., Hellhammer, D., 2004. Increased responsiveness of the hypothalamus-pituitary-adrenal (HPA) axis to stress in newborns with atopic disposition. Psychoneuroendocrinology 29, 705-711.

Chida, Y., Steptoe, A., 2009. Cortisol awakening response and psychosocial factors: a systematic review and meta-analysis. Biol Psychol 80, 265-278.

Clow, A., Hucklebridge, F., Stalder, T., Evans, P., Thorn, L., 2010. The cortisol awakening response: more than a measure of HPA axis function. Neurosci Biobehav Rev 35, 97-103.

Clow, A., Thorn, L., Evans, P., Hucklebridge, F., 2004. The awakening cortisol response: methodological issues and significance. Stress 7, 29-37.

Dickerson, S.S., Kemeny, M.E., 2004. Acute stressors and cortisol responses: a theoretical integration and synthesis of laboratory research. Psychol Bull 130, 355-391.

Dockray, S., Bhattacharyya, M.R., Molloy, G.J., Steptoe, A., 2008. The cortisol awakening response in relation to objective and subjective measures of waking in the morning. Psychoneuroendocrinology 33, 77-82.

Edwards, S., Clow, A., Evans, P., Hucklebridge, F., 2001. Exploration of the awakening cortisol response in relation to diurnal cortisol secretory activity. Life Sci 68, 2093-2103. Felt, B.T., Mollen, E., Diaz, S., Renaud, E., Zeglis, M., Wheatcroft, G., Mendelow, D., 2000. Behavioral interventions reduce infant distress at immunization. Arch Pediatr Adolesc Med $154,719-724$.

Fries, E., Dettenborn, L., Kirschbaum, C., 2009. The cortisol awakening response (CAR): facts and future directions. Int J Psychophysiol 72, 67-73. 
Galland, B.C., Taylor, B.J., Elder, D.E., Herbison, P., 2012. Normal sleep patterns in infants and children: a systematic review of observational studies. Sleep Med Rev 16, 213-222. Goldberg, S., Levitan, R., Leung, E., Masellis, M., Basile, V.S., Nemeroff, C.B., Atkinson, L., 2003. Cortisol concentrations in 12- to 18-month-old infants: stability over time, location, and stressor. Biol Psychiatry 54, 719-726.

Groschl, M., Rauh, M., Dorr, H.G., 2003. Circadian rhythm of salivary cortisol, 17alphahydroxyprogesterone, and progesterone in healthy children. Clin Chem 49, 1688-1691. Gunnar, M.R., Brodersen, L., Krueger, K., Rigatuso, J., 1996. Dampening of adrenocortical responses during infancy: normative changes and individual differences. Child development $67,877-889$.

Gunnar, M.R., Talge, N.M., Herrera, A., 2009. Stressor paradigms in developmental studies: what does and does not work to produce mean increases in salivary cortisol. Psychoneuroendocrinology 34, 953-967.

Gustafsson, P.E., Janlert, U., Theorell, T., Hammarstrom, A., 2010. Is body size at birth related to circadian salivary cortisol levels in adulthood? Results from a longitudinal cohort study. BMC Public Health 10, 346.

Hellhammer, D.H., Wust, S., Kudielka, B.M., 2009. Salivary cortisol as a biomarker in stress research. Psychoneuroendocrinology 34, 163-171.

Hellhammer, J., Fries, E., Schweisthal, O.W., Schlotz, W., Stone, A.A., Hagemann, D., 2007. Several daily measurements are necessary to reliably assess the cortisol rise after awakening: state- and trait components. Psychoneuroendocrinology 32, 80-86.

Jansen, J., Beijers, R., Riksen-Walraven, M., de Weerth, C., 2010. Cortisol reactivity in young infants. Psychoneuroendocrinology 35, 329-338.

Jessop, D.S., Turner-Cobb, J.M., 2008. Measurement and meaning of salivary cortisol: a focus on health and disease in children. Stress 11, 1-14. 
Jones, A., Godfrey, K.M., Wood, P., Osmond, C., Goulden, P., Phillips, D.I., 2006. Fetal growth and the adrenocortical response to psychological stress. The Journal of clinical endocrinology and metabolism 91, 1868-1871.

Jones, A., Osmond, C., Godfrey, K.M., Phillips, D.I., 2011. Evidence for developmental programming of cerebral laterality in humans. PLoS One 6, e17071.

Kajantie, E., Phillips, D.I., 2006. The effects of sex and hormonal status on the physiological response to acute psychosocial stress. Psychoneuroendocrinology 31, 151-178.

Kiess, W., Meidert, A., Dressendorfer, R.A., Schriever, K., Kessler, U., Konig, A., Schwarz, H.P., Strasburger, C.J., 1995. Salivary cortisol levels throughout childhood and adolescence: relation with age, pubertal stage, and weight. Pediatric research 37, 502-506.

Kudielka, B.M., Hawkley, L.C., Adam, E.K., Cacioppo, J.T., 2007. Compliance with ambulatory saliva sampling in the chicago health, aging, and social relations study and associations with social support. Ann Behav Med 34, 209-216.

Kudielka, B.M., Kirschbaum, C., 2003. Awakening cortisol responses are influenced by health status and awakening time but not by menstrual cycle phase.

Psychoneuroendocrinology 28, 35-47.

Kudielka, B.M., Kirschbaum, C., 2005. Sex differences in HPA axis responses to stress: a review. Biol Psychol 69, 113-132.

Kudielka, B.M., Wust, S., 2010. Human models in acute and chronic stress: assessing determinants of individual hypothalamus-pituitary-adrenal axis activity and reactivity. Stress $13,1-14$.

Lucas, A., 1991. Programming by early nutrition in man. Ciba Found Symp 156, 38-50; discussion 50-35.

Lupien, S.J., McEwen, B.S., Gunnar, M.R., Heim, C., 2009. Effects of stress throughout the lifespan on the brain, behaviour and cognition. Nat Rev Neurosci 10, 434-445. 
Magnano, C.L., Diamond, E.J., Gardner, J.M., 1989. Use of salivary cortisol measurements in young infants: a note of caution. Child Dev 60, 1099-1101.

Matthews, S.G., 2002. Early programming of the hypothalamo-pituitary-adrenal axis. Trends Endocrinol Metab 13, 373-380.

Meaney, M.J., Szyf, M., Seckl, J.R., 2007. Epigenetic mechanisms of perinatal programming of hypothalamic-pituitary-adrenal function and health. Trends Mol Med 13, 269-277.

Meinlschmidt, G., Heim, C., 2005. Decreased cortisol awakening response after early loss experience. Psychoneuroendocrinology 30, 568-576.

Mesiano, S., Jaffe, R.B., 1997. Developmental and functional biology of the primate fetal adrenal cortex. Endocr Rev 18, 378-403.

Netherton, C., Goodyer, I., Tamplin, A., Herbert, J., 2004. Salivary cortisol and dehydroepiandrosterone in relation to puberty and gender. Psychoneuroendocrinology 29, $125-140$.

O'Connor, T.G., Bergman, K., Sarkar, P., Glover, V., 2012. Prenatal cortisol exposure predicts infant cortisol response to acute stress. Developmental psychobiology. doi: 10.1002/dev.21007.

Oken, E., Kleinman, K.P., Rich-Edwards, J., Gillman, M.W., 2003. A nearly continuous measure of birth weight for gestational age using a United States national reference. BMC Pediatr 3, 6 .

Osmond, C., Barker, D.J., 2000. Fetal, infant, and childhood growth are predictors of coronary heart disease, diabetes, and hypertension in adult men and women. Environ Health Perspect 108 Suppl 3, 545-553.

Osterholm, E.A., Hostinar, C.E., Gunnar, M.R., 2012. Alterations in stress responses of the hypothalamic-pituitary-adrenal axis in small for gestational age infants.

Psychoneuroendocrinology. doi: 10.1016/j.psyneuen.2012.03.005. 
Phillips, D.I., 2007. Programming of the stress response: a fundamental mechanism underlying the long-term effects of the fetal environment? J Intern Med 261, 453-460. Phillips, D.I., Jones, A., 2006. Fetal programming of autonomic and HPA function: do people who were small babies have enhanced stress responses? J Physiol 572, 45-50.

Phillips, D.I., Walker, B.R., Reynolds, R.M., Flanagan, D.E., Wood, P.J., Osmond, C., Barker, D.J., Whorwood, C.B., 2000. Low birth weight predicts elevated plasma cortisol concentrations in adults from 3 populations. Hypertension 35, 1301-1306.

Price, D.A., Close, G.C., Fielding, B.A., 1983. Age of appearance of circadian rhythm in salivary cortisol values in infancy. Arch Dis Child 58, 454-456.

Pruessner, J.C., Wolf, O.T., Hellhammer, D.H., Buske-Kirschbaum, A., von Auer, K., Jobst, S., Kaspers, F., Kirschbaum, C., 1997. Free cortisol levels after awakening: a reliable biological marker for the assessment of adrenocortical activity. Life Sci 61, 2539-2549. Reynolds, R.M., Walker, B.R., Syddall, H.E., Andrew, R., Wood, P.J., Whorwood, C.B., Phillips, D.I., 2001. Altered control of cortisol secretion in adult men with low birth weight and cardiovascular risk factors. J Clin Endocrinol Metab 86, 245-250.

Santiago, L.B., Jorge, S.M., Moreira, A.C., 1996. Longitudinal evaluation of the development of salivary cortisol circadian rhythm in infancy. Clin Endocrinol (Oxf) 44, 157-161.

Sapolsky, R.M., Romero, L.M., Munck, A.U., 2000. How do glucocorticoids influence stress responses? Integrating permissive, suppressive, stimulatory, and preparative actions. Endocr Rev 21, 55-89.

Schapiro, S., Geller, E., Eiduson, S., 1962. Neonatal adrenal cortical response to stress and vasopressin. Proc Soc Exp Biol Med 109, 937-941.

Schmidt, M.V., Enthoven, L., van der Mark, M., Levine, S., de Kloet, E.R., Oitzl, M.S., 2003. The postnatal development of the hypothalamic-pituitary-adrenal axis in the mouse. Int J Dev Neurosci 21, 125-132. 
Strazdins, L., Meyerkort, S., Brent, V., D'Souza, R.M., Broom, D.H., Kyd, J.M., 2005. Impact of saliva collection methods on SIgA and cortisol assays and acceptability to participants. $\mathrm{J}$ Immunol Methods 307, 167-171.

Tegethoff, M., Greene, N., Olsen, J., Meyer, A.H., Meinlschmidt, G., 2010a. Maternal psychosocial adversity during pregnancy is associated with length of gestation and offspring size at birth: evidence from a population-based cohort study. Psychosom Med 72, 419-426. Tegethoff, M., Greene, N., Olsen, J., Meyer, A.H., Meinlschmidt, G., 2010b. Maternal psychosocial stress during pregnancy and placenta weight: evidence from a national cohort study. PLoS One 5, e14478.

Tegethoff, M., Greene, N., Olsen, J., Schaffner, E., Meinlschmidt, G., 2011a. Stress during pregnancy and offspring pediatric disease: A National Cohort Study. Environmental health perspectives $119,1647-1652$.

Tegethoff, M., Greene, N., Olsen, J., Schaffner, E., Meinlschmidt, G., 2012. Inhaled glucocorticoids during pregnancy and offspring pediatric diseases: a national cohort study. Am J Respir Crit Care Med 185, 557-563.

Tegethoff, M., Pryce, C., Meinlschmidt, G., 2009. Effects of intrauterine exposure to synthetic glucocorticoids on fetal, newborn, and infant hypothalamic-pituitary-adrenal axis function in humans: a systematic review. Endocr Rev 30, 753-789.

Tegethoff, M., Raul, J.S., Jamey, C., Khelil, M.B., Ludes, B., Meinlschmidt, G., 2011 b. Dehydroepiandrosterone in nails of infants: a potential biomarker of intrauterine responses to maternal stress. Biol Psychol 87, 414-420.

Tikotzky, L., G, D.E.M., Har-Toov, J., Dollberg, S., Bar-Haim, Y., Sadeh, A., 2010. Sleep and physical growth in infants during the first 6 months. J Sleep Res 19, 103-110. Tollenaar, M.S., Beijers, R., Jansen, J., Riksen-Walraven, J.M., de Weerth, C., 2011. Maternal prenatal stress and cortisol reactivity to stressors in human infants. Stress 14, 53-65. 
Van Cauter, E., Leproult, R., Kupfer, D.J., 1996. Effects of gender and age on the levels and circadian rhythmicity of plasma cortisol. J Clin Endocrinol Metab 81, 2468-2473.

Wang, M.L., Dorer, D.J., Fleming, M.P., Catlin, E.A., 2004. Clinical outcomes of near-term infants. Pediatrics 114, 372-376.

Ward, A.M., Syddall, H.E., Wood, P.J., Chrousos, G.P., Phillips, D.I., 2004. Fetal programming of the hypothalamic-pituitary-adrenal (HPA) axis: low birth weight and central HPA regulation. J Clin Endocrinol Metab 89, 1227-1233.

Watamura, S.E., Donzella, B., Kertes, D.A., Gunnar, M.R., 2004. Developmental changes in baseline cortisol activity in early childhood: relations with napping and effortful control. Developmental psychobiology 45, 125-133.

Weitzman, E.D., Fukushima, D., Nogeire, C., Roffwarg, H., Gallagher, T.F., Hellman, L., 1971. Twenty-four hour pattern of the episodic secretion of cortisol in normal subjects. J Clin Endocrinol Metab 33, 14-22.

White, B.P., Gunnar, M.R., Larson, M.C., Donzella, B., Barr, R.G., 2000. Behavioral and physiological responsivity, sleep, and patterns of daily cortisol production in infants with and without colic. Child Dev 71, 862-877.

Wilhelm, I., Born, J., Kudielka, B.M., Schlotz, W., Wust, S., 2007. Is the cortisol awakening rise a response to awakening? Psychoneuroendocrinology 32, 358-366.

Wust, S., Entringer, S., Federenko, I.S., Schlotz, W., Hellhammer, D.H., 2005. Birth weight is associated with salivary cortisol responses to psychosocial stress in adult life.

Psychoneuroendocrinology 30, 591-598.

Wust, S., Wolf, J., Hellhammer, D.H., Federenko, I., Schommer, N., Kirschbaum, C., 2000. The cortisol awakening response - normal values and confounds. Noise Health 2, 79-88. 
Tegethoff et al.: CAR in infants

\section{Figure captions}

Figure 1: Flowchart of study participants.

Figure 2: Cortisol awakening responses. Cortisol concentrations at 0 and +30 minutes after awakening, on day 1 and day 2. SEM, standard error of the mean. 


\section{Tables}

Table 1: Characteristics of mother-infant pairs $(N=64)$.

Continuous Variables: Mean (standard deviation; range) [number of mother-infant pairs with respective information unknown]

\begin{tabular}{lr}
\hline Mother's age at birth (years) & $33.8(4.6 ; 21-42)[0]$ \\
Mother's height before pregnancy $(\mathrm{cm})$ & $168(6.5 ; 154-181)[0]$ \\
Mother's weight before pregnancy $(\mathrm{kg})$ & $61.7(8.4 ; 48-89)[1]$ \\
Offspring's birth weight $(\mathrm{g})$ & $3519(520.3 ; 2315-4550)[0]$ \\
Offspring's body length at birth (cm) & $50.5(2.6 ; 44-60)[0]$ \\
Length of gestation (weeks) & $40.1(1.7 ; 35-44)[0]$ \\
\hline
\end{tabular}

Continuous Variables: Median (range) [number of mother-infant pairs without information on cortisol concentrations from the respective sampling day (or both days for the interval between sampling days) that was entered in the analyses]

Offspring's age at time of saliva collection at day 1 (days) $58(24-165)[2]$

Offspring's age at time of saliva collection at day 2 (days) $66(25-171)[5]$

Interval between sampling days (days)

$1(1-31)[7]$

\begin{tabular}{|c|c|}
\hline & \\
\hline \multicolumn{2}{|l|}{ Mode of delivery } \\
\hline Spontaneous birth & $45(70.3)$ \\
\hline Forceps delivery & $4(6.3)$ \\
\hline Cesarean section & $15(23.4)$ \\
\hline Unknown & $\begin{array}{ll}0 & (0)\end{array}$ \\
\hline \multicolumn{2}{|l|}{ Parity } \\
\hline Primipara & $29(45.3)$ \\
\hline Multipara & $35(54.7)$ \\
\hline Unknown & $\begin{array}{ll}0 & (0)\end{array}$ \\
\hline \multicolumn{2}{|l|}{ Offspring sex } \\
\hline Male & $30(46.9)$ \\
\hline Female & $34(53.1)$ \\
\hline Unknown & $0 \quad(0)$ \\
\hline \multicolumn{2}{|l|}{ Socioeconomic status } \\
\hline Low & $2(3.0)$ \\
\hline Medium & $43(67.2)$ \\
\hline High & $18(28.1)$ \\
\hline Unknown & 1 (1.6) \\
\hline \multicolumn{2}{|c|}{ Smoking during pregnancy } \\
\hline Yes & $4(6.3)$ \\
\hline No & $60(93.7)$ \\
\hline Unknown & $0 \quad(0)$ \\
\hline
\end{tabular}


Mother-infant pairs assessed for eligibility $(N=107)$

No saliva sample provided

$(N=25 ; 23.4 \%)$

At least one saliva sample provided

( $N=82 ; 76.6 \%$ of those assessed for eligibility)

All subjects providing their saliva samples later than six months postpartum or at unknown age ( $N=5 ; 6.1 \%$ of those providing at least one saliva sample) or subjects with not more than one sample provided on each sampling day $(N=13$; $15.9 \%$ of those providing at least one saliva sample).

Included in analyses: $N=64$ (78.0\% of those providing at least one saliva sample), contributing a total of 242 saliva samples that were collected within the first six months of life, with samples from sampling day 2 collected not later than 45 days after sampling day 1 . 


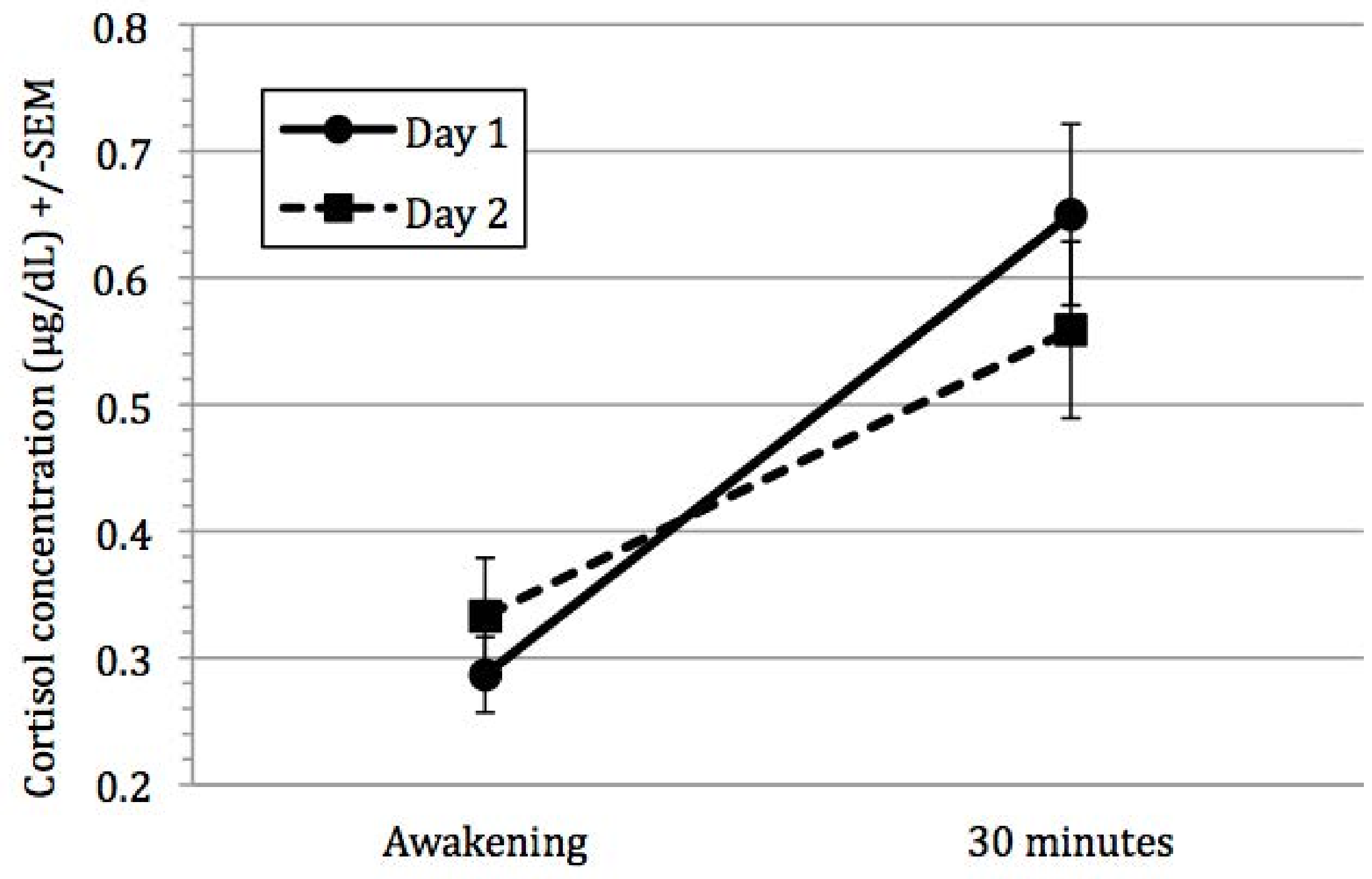


Role of funding source: This project was financed by the German National Academic Foundation and the Research Foundation of the University of Basel (to MT), and the Swiss National Science Foundation (SNSF), projects no. PZ00P1_137023 (to M.T.), as well as no. 100014_135328 and no. 51A240-104890 (to GM). The funding sources had no role in study design; in the collection, analysis and interpretation of data; in the writing of the report; and in the decision to submit the paper for publication.

Conflict of interest: The authors declare that they have no conflicts of interest.

Contributors: MT and GM designed the study and wrote the protocol; MT, NK and GM managed the literature searches and analyses; MT, AM and GM undertook the statistical analysis; MT and NK wrote the first draft of the manuscript. All authors contributed to and have approved the final manuscript.

Acknowledgments: We thank Simone Briner Meier, Sigrid Falk, Melanie Knabe, Melanie Kruegel, Dominique Moser, and Lena Temp for their valuable help with the project. 